\title{
Revisiting the Complexity of Hardware Cache Coherence and Some Implications
}

\author{
RAKESH KOMURAVELLI and SARITA V. ADVE, University of Illinois at Urbana-Champaign \\ CHING-TSUN CHOU, Intel Corporation
}

Cache coherence is an integral part of shared-memory systems but is also widely considered to be one of the most complex parts of such systems. Much prior work has addressed this complexity and the verification techniques to prove the correctness of hardware coherence. Given the new multicore era with increasing number of cores, there is a renewed debate about whether the complexity of hardware coherence has been tamed or whether it should be abandoned in favor of software coherence. This article revisits the complexity of hardware cache coherence by verifying a publicly available, state-of-the-art implementation of the widely used MESI protocol, using the Mur $\varphi$ model checking tool. To our surprise, we found six bugs in this protocol, most of which were hard to analyze and took several days to fix. To compare the complexity, we also verified the recently proposed DeNovo protocol, which exploits disciplined software programming models. We found three relatively easy to fix bugs in this less mature protocol. After fixing these bugs, our verification experiments showed that, compared to DeNovo, MESI had 15X more reachable states leading to a $20 \mathrm{X}$ increase in verification (model checking) time. Although we were eventually successful in verifying the protocols, the tool required making several simplifying assumptions (e.g., two cores, one address). Our results have several implications: (1) they indicate that hardware coherence protocols remain complex; (2) they reinforce the need for protocol designers to embrace formal verification tools to demonstrate correctness of new protocols and extensions; (3) they reinforce the need for formal verification tools that are both scalable and usable by non-expert; and (4) they show that a system based on hardware-software co-design can offer a simpler approach for cache coherence, thus reducing the overall verification effort and allowing verification of more detailed models and protocol extensions that are otherwise limited by computing resources.

Categories and Subject Descriptors: B.3.2 [Design Styles]: Cache memories; B.3.2 [Design Styles]: Shared memory; B.3.3 [Performance Analysis and Design Aids]: Formal models; C.0 [General]: Modeling of computer architecture

General Terms: Design, Performance, Verification

Additional Key Words and Phrases: Cache coherence, multicore, shared memory, software-hardware co-design, protocol verification, model checking

This work was supported in part by Intel and Microsoft through the Universal Parallel Computing Research Center (UPCRC) at Illinois, by Intel through the Illinois/Intel Parallelism Center at Illinois, by the National Science Foundation under grants CCF-1018796 and CCF-1302641, and by the Center for Future Architectures Research (C-FAR), one of six centers of STARnet, a Semiconductor Research Corporation program sponsored by MARCO and DARPA. Rakesh Komuravelli was also supported by a Qualcomm Innovation Fellowship.

Authors' addresses: R. Komuravelli and S. Adve, 201 N Goodwin Ave, Urbana, IL 61801 USA; emails: \{komurav1, sadve\}@illinois.edu; C.-T. Chou, Intel Corporation 3600 Juliette Lane Santa Clara, CA 95054 USA; ching-tsun.chou@intel.com.

Permission to make digital or hard copies of part or all of this work for personal or classroom use is granted without fee provided that copies are not made or distributed for profit or commercial advantage and that copies show this notice on the first page or initial screen of a display along with the full citation. Copyrights for components of this work owned by others than ACM must be honored. Abstracting with credit is permitted. To copy otherwise, to republish, to post on servers, to redistribute to lists, or to use any component of this work in other works requires prior specific permission and/or a fee. Permissions may be requested from Publications Dept., ACM, Inc., 2 Penn Plaza, Suite 701, New York, NY 10121-0701 USA, fax +1 (212) 869-0481, or permissions@acm.org.

(c) 2014 ACM 1544-3566/2014/12-ART37 $\$ 15.00$

DOI: http://dx.doi.org/10.1145/2663345 


\section{ACM Reference Format:}

Rakesh Komuravelli, Sarita V. Adve, and Ching-Tsun Chou. 2014. Revisiting the complexity of hardware cache coherence and some implications. ACM Trans. Architec. Code Optim. 11, 4, Article 37 (December 2014), 22 pages.

DOI: http://dx.doi.org/10.1145/2663345

\section{INTRODUCTION}

Shared memory continues to be one of the most widely used parallel programming models due to the advantages offered by a global address space. Today's multicores directly support shared memory and form the heart of a variety of computing systems, ranging from smartphones to data centers to the largest supercomputers. One of the main challenges of shared memory hardware is to keep caches coherent, and this is usually addressed by hardware cache coherence protocols. Traditionally, a major problem with such protocols has been that they have been extremely complex, and implementing bug-free protocols has been a challenge [Abts et al. 2003]. Increasing on-chip core counts in manycores exacerbates this problem because it demands moving to variants of directory-based protocols. Methods like trace-driven or other simulationbased testing are insufficient for ensuring the correctness of such complex protocols since they often do not hit the difficult corner cases. Formal verification techniques have therefore been proposed and deployed to prove the correctness of these protocols [Abts et al. 2000; Burckhardt et al. 2005; Gjessing et al. 1989; McMillan and J. 1991; Nanda and Bhuyan 1992; Park and Dill 1995; Pong et al. 1998].

Over the past several decades, there has been much experience accumulated in both designing cache coherence protocols and building formal methods based tools to verify them. Nevertheless, as the number of cores on a chip grows, the power, performance, and area inefficiencies of hardware cache coherence (e.g., directory storage, indirection through the directory, invalidation and acknowledgment messages, false sharing, cache line based granularities, and so on) are potential obstacles to scalability. To reduce these inefficiencies, researchers continue to propose enhancements to basic coherence protocols - these enhancements typically come at the cost of even further complexity [Zebchuk et al. 2009; Zhao et al. 2013].

There is a debate in the community about whether we have tamed the complexity of hardware cache coherence enough to continue building increasingly larger hardware cache coherent systems or whether we must abandon hardware coherence in favor of other alternatives. Thus, on the one hand, companies like Tilera have built hardware cache coherent systems with as many as 64 cores, making the resulting familiar programming model a key differentiator for their product line. A recent position paper makes the case that hardware coherence is here to stay [Martin et al. 2012]. Other researchers continue to extend current protocols in new ways that make them even more complex [Aisopos and Peh 2011]. On the other hand, Intel has recently built a 48-core Single-Chip Cloud Computer (SCC) [IntelSCC 2009] that abandons hardware cache coherence, replacing it with software managed caches and their attendant programming complexity. Academic and industry researchers have also proposed systems that eliminate dependence on hardware coherence in various ways [Borkar 2011; Choi et al. 2011; Kelm et al. 2009; Lin et al. 2012].

In this article, we revisit the complexity of hardware cache coherence by asking the following questions. Has our experience with hardware cache coherence in the past few decades enabled us to conquer its complexity? If not, what are the implications for future system design?

To answer these questions, ideally, we would use an industry-strength cache coherence protocol specification and quantitative metrics for its complexity. Given the proprietary nature of industry protocols, we instead use a state-of-the-art, mature, publicly available protocol, the MESI protocol implemented in the Wisconsin GEMS simulation 
suite (version 2.1.1) [Martin et al. 2005]. ${ }^{1}$ GEMS was released in 2005 and remains a widely used simulation package in the computer architecture community. Unfortunately, however, there are no currently accepted quantitative metrics to measure complexity. Instead, we report on our experiences verifying the GEMS MESI protocol using the Mur $\varphi$ model checking tool (version 3.1, slightly modified to exploit 64-bit machines) [Dill et al. 1992; Ip and Dill 1993, 1996]. Although more advanced verification techniques exist, we chose $\operatorname{Mur} \varphi$ for its easy-to-use interface and robustness. Mur $\varphi$ has also been the tool of choice for many hardware cache-related studies [Abts et al. 2000; Burckhardt et al. 2005; Park and Dill 1995; Pong et al. 1998; Zhang et al. 2010].

Our results from applying $\operatorname{Mur} \varphi$ to the GEMS MESI protocol were surprising. We found six bugs in the protocol, even though it is a mature protocol used by a large number of architecture researchers. More significantly, some of these bugs involved subtle races and took several days to debug and fix. We contacted the developers of the GEMS simulation team with our bug findings in 2011. They had seen one of the six bugs, bug \#4 in Section 4.1, but were surprised by the other bugs. Some of these bugs were also present in the GEM5 simulator [Binkert et al. 2011], an extension to the GEMS simulator that incorporates the M5 CPU core simulator, at that time. After we showed our fixes, the GEMS group fixed the bugs and released new patches. These fixes needed the addition of multiple new state transitions and extra buffer space for stalling requests in the protocol. After fixing the protocol, we were able to successfully run $\operatorname{Mur} \varphi$, but the tool required making several simplifying assumptions typical of such tools today (e.g., two cores, single cache line per core, and one address). With these assumptions, the verification process explored 1,257,500 reachable states and took 173 seconds using the default breadth-first search option of Mur $\varphi$. We ran out of memory on our machine (with $32 \mathrm{~GB}$ of RAM) when we tried to model a system containing two memory addresses.

Our conclusion from this experience is that hardware cache coherence protocols still remain difficult to design, understand, and prove correct. Although formal verification techniques have been embraced by industry, their use is not yet common practice for architecture researchers. Our results reinforce the need to use formal verification tools to assess the correctness and complexity of coherence protocols. Conversely, our work also motivates the need for verification tools that are scalable to larger systems and usable by non-expert.

An extreme solution to the hardware complexity problem is represented by the Intel SCC [IntelSCC 2009]. The SCC eliminates the hardware complexity of cache coherence but imposes significant programming complexity due to the requirement of managing the caches in software. Recently, we proposed an alternate method, called DeNovo, that is based on a tighter hardware/software co-design approach [Choi et al. 2011]. For easier programmability, this approach espouses disciplined shared-memory programming languages that eliminate data races by design, provide determinism-by-default, and provide safe nondeterminism only when explicitly requested. With a guarantee of datarace-freedom from software, DeNovo is able to avoid races in hardware and the resulting transient states and their complexity. The simplicity of the protocol, coupled with the disciplined software, provides performance and power advantages to DeNovo as well; furthermore, various enhancements (optimizations) can be incorporated without adding new states or transitions [Choi et al. 2011]. We report our experiences with using $\operatorname{Mur} \varphi$ to verify the DeNovo protocol and compare them with the MESI experiences.

To verify DeNovo, we first extended the canonical coherence protocol modeling technique in $\operatorname{Mur} \varphi$ to model the software constraints that DeNovo hardware expects. In

\footnotetext{
${ }^{1}$ We chose MESI over a more complicated MOESI from GEMS because the GEMS web site says that the CMP version of the latter has bugs. Although we specifically study the MESI protocol in this article, we believe our insights apply to other protocols, such as MOESI, as well.
} 
particular, this extension is able to guarantee the data-race-free property and a disciplined parallel phase behavior. Using this extension, we verified the base DeNovo protocol. Despite DeNovo's immaturity, we found only three bugs in the implementation. Furthermore, these bugs were simple to fix and turned out to be mistakes in translating the high level description of the protocol into the implementation (i.e., their solutions were already present in the internal high level description of the protocol). After fixing the bugs, we found that, compared to MESI, Mur $\varphi$ had to explore 15X fewer reachable states for DeNovo, taking $20 \mathrm{X}$ less time to fully model check DeNovo. It is difficult to define a metric to quantify the relative verification complexity of coherence protocols; nevertheless, our results demonstrate that hardware-software co-designed approaches like DeNovo can lead to much simpler protocols than conventional hardware cache coherence (while providing an easy programming model, extensibility, and competitive or better performance [Choi et al. 2011]). ${ }^{2}$

Overall, this article shows that despite the accumulated experience in designing hardware cache coherence, these protocols remain complex. It reinforces the need to use formal verification by protocol designers, motivating scalable and easy-to-use tools for non-experts. Finally, the article motivates hardware-software co-designed protocols that are much simpler to design and verify than pure hardware coherence and that exploit simpler programming models.

\section{COMPLEXITY OF CACHE COHERENCE}

Cache coherence is usually defined as a mechanism that ensures writes to the same memory location appear in the same order to all cores. In this section, we discuss two directory based protocols, the MESI protocol, which is based on a state-of-the-art implementation obtained from the publicly available GEMS simulator [Martin et al. 2005], and the recent DeNovo protocol [Choi et al. 2011], which is based on our own implementation. Without loss of generality, we assume a multicore system with $n$ cores, private $L 1$ caches, a shared $L 2$ cache, and a general (nonbus, unordered) interconnect on chip.

\subsection{MESI}

MESI, also known as the Illinois protocol [Papamarcos and Patel 1984], stands for Modified (locally modified and no other cache has a copy), Exclusive (unmodified and no other cache has a copy), Shared (unmodified and some other caches may have a copy), and Invalid. Over the MSI protocol, the Exclusive state has the added advantage of avoiding invalidation traffic on write hits. For scalability, we assume a directory protocol [Lenoski et al. 1992]. Given our shared (inclusive) L2 cache-based multicore, we assume a directory entry per $L 2$ cache line, referred to as an in-cache directory [Censier and Feautrier 1978]. We use L2 and directory interchangeably.

Figure 1 shows the simple textbook state transition diagram for an $L 1$ cache with the MESI protocol. The $L 2$ cache also has four (textbook) states: L1_Modified (modified in a local L1), L2_Modified (modified at L2 and not present in any L1), Shared (valid data at $L 2$ and present in one or more L1s), and Invalid. It also has a dirty bit (set on receiving a writeback from $L 1$ ) that indicates whether data is dirty or not. When in Shared state, the L2/directory contains the up-to-date copy of the cache block data along with a list of sharers. On a read miss request, the directory services the request

\footnotetext{
${ }^{2}$ The paper proposing DeNovo [Choi et al. 2011] showed that the basic DeNovo protocol is performancecompetitive with MESI, and its simplicity enables optimizations that significantly improved performance and network traffic (and thereby energy) [Choi et al. 2011]. That paper focused on performance, but as evidence of DeNovo's simplicity, it also briefly summarized some of the key results of this article. That paper only gave the number of bugs found in each protocol, the number of states explored, and the verification times. It did not describe the nature of the bugs in MESI or DeNovo, the fixes involved, or the modification to standard Mur $\varphi$ modeling required to verify a hardware/software co-designed protocol like DeNovo.
} 


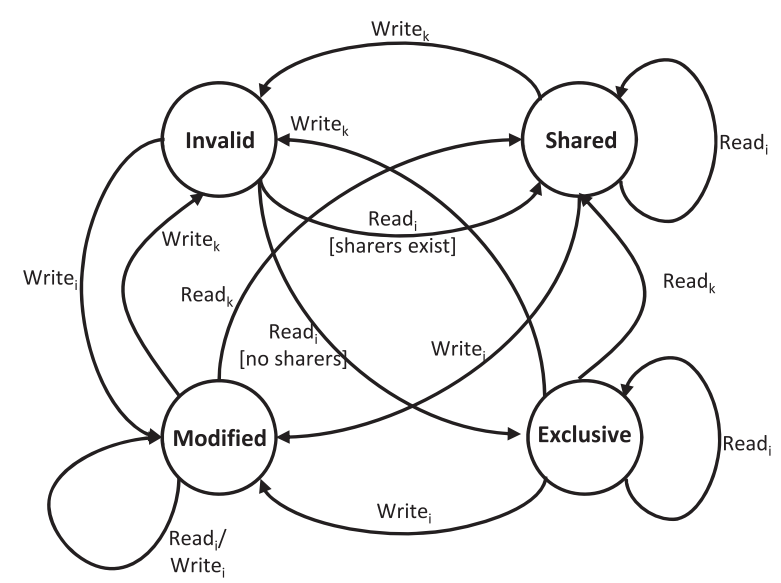

Fig. 1. Textbook state transition diagram for $L 1$ cache of core $i$ for the MESI protocol. $\operatorname{Read}_{i}=\operatorname{read}$ from core $\mathrm{i}, \operatorname{Read}_{k}=$ read from another core $k$.

if it has the up-to-date copy, or else it forward the request to the core that has the exclusive or modified copy. On a write miss or upgrade request, the directory sends invalidation requests to all the sharers (if any). If a request misses in the $L 2$, the block is fetched from the main memory.

In reality, this seemingly simple protocol is a lot more complex. The hardware implementation of the protocol has many transient states in addition to the four states just described. These transient states lead to various subtle races and are the root cause of complexity in the protocol. We now illustrate the need for transient states with an example. Figure 2(a) shows a code snippet with two parallel phases accessing a shared variable $A .^{3}$

In the first phase, cores $P 2$ through $P n$ read the shared variable $A$, and, in the second phase, core $P 1$ writes to $A$. In this example, we focus mainly on the state transitions related to this write by core $P 1$.

Figure 2(b) shows the timeline of the state transitions at both the individual $L 1$ s and the $L 2$. Figure 2(c) and (d) show the state transition table for $L 1$ and $L 2$, respectively, for the states encountered in this example. The names of the states and the events are taken directly from the GEMS implementation. At the beginning of the second phase, cores $P 2$ through $P n$ are in Shared state and they are recorded in the sharer list at the directory. On receiving the write request, $L 1_{P 1}$ issues a $G E T X$ request to $L 2$ and transitions to the first transient state, $I M$, where it awaits the data response from $L 2$. $L 2$, on receiving the $G E T X$ request, sends the data response (including the number of $A c k \mathrm{~s}$ to expect) to $L 1_{P 1}$; sends invalidation requests to all the sharers $\left(L 1_{P 2}\right.$ through $L 1_{P n}$ ); and then transitions to a transient state, $S S_{-} M B$, where it awaits an unblock message from the original requestor indicating the completion of the request. $L 1_{P 1}$, on receiving the data response from $L 2$, transitions to the second transient state, $S M$, where it waits for all the Ack messages from the sharers. Every sharer, then, on receiving the invalidation message from $L 2$, transitions to the Invalid state and responds directly to the requestor, $L 1_{P 1}$, with an Ack message. When $L 1_{P 1}$ receives the last Ack message (Ack_all event), it transitions to the Modified state and unblocks L2 by sending an Exclusive_Unblock message. Other cases (e.g., some of the Acks arriving at $L 1_{P 1}$ before it receives the data response from $L 2$, etc.) are covered in the state

\footnotetext{
${ }^{3}$ For the purpose of this article, we assume a phase is a computation between two successive barriers (e.g., a parallel for loop).
} 


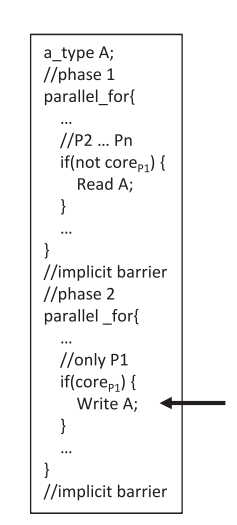

(a) Example parallel code

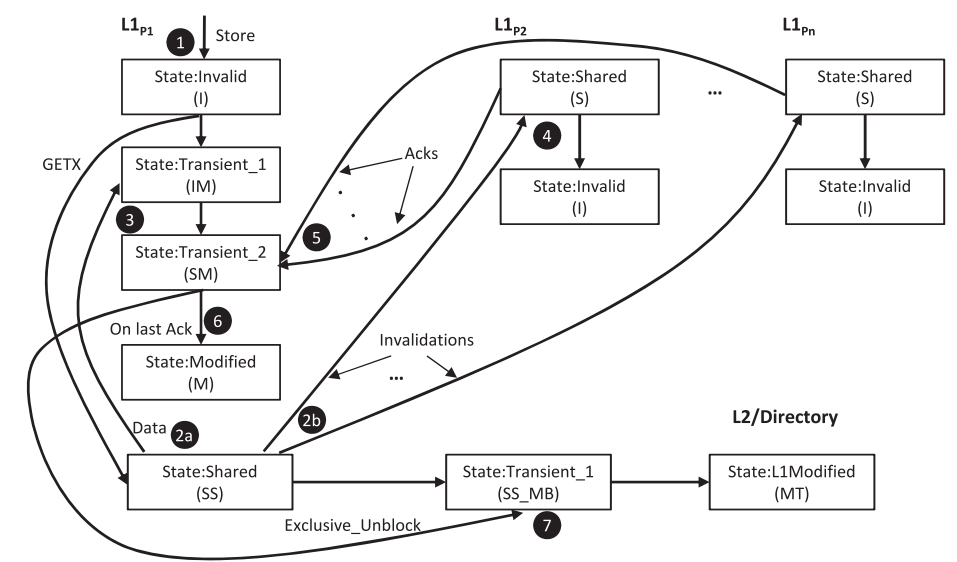

(b) Timeline of state transitions at $\mathrm{L} 1$ and $\mathrm{L} 2$

\begin{tabular}{|c|c|c|c|c|c|}
\hline State/Event & Write_i & Ack & Ack_all & Data & Data_Ack_all \\
\hline $\begin{array}{c}\text { Invalid } \\
\text { (I) }\end{array}$ & $\begin{array}{c}\text { Go to } \\
\text { Transient_1; } \\
\text { GETX to L2; }\end{array}$ & $\mathrm{X}$ & $\mathrm{X}$ & $\mathrm{X}$ & $\mathrm{X}$ \\
\hline $\begin{array}{c}\text { Transient_1 } \\
\text { (IM) }\end{array}$ & Keep pending; & $\begin{array}{c}\text { Update } \\
\text { Ack count; }\end{array}$ & $\mathrm{X}$ & $\begin{array}{c}\text { Update } \\
\text { Ack count; } \\
\text { Go to } \\
\text { Transient_2; }\end{array}$ & $\begin{array}{c}\text { Go to Modified; } \\
\text { Exclusive_Unblock } \\
\text { to L2; }\end{array}$ \\
\hline $\begin{array}{c}\text { Transient_2 } \\
\text { (SM) }\end{array}$ & Keep pending; & $\begin{array}{c}\text { Update } \\
\text { Ack count; }\end{array}$ & $\begin{array}{c}\text { Go to Modified; } \\
\text { Exclusive_Unblock } \\
\text { to L2; }\end{array}$ & $\mathrm{X}$ & $\mathrm{X}$ \\
\hline
\end{tabular}

(c) L1 state transition table

\begin{tabular}{|c|c|c|}
\hline State/Event & L1_GETX & Exclusive_Unblock \\
\hline $\begin{array}{c}\text { Shared } \\
\text { (SS) }\end{array}$ & $\begin{array}{c}\text { Go to Transient_1; } \\
\text { Ack to requestor; } \\
\text { Inv to sharers; }\end{array}$ & $\mathrm{X}$ \\
\hline $\begin{array}{c}\text { Transient_1 } \\
\text { (SS_MB) }\end{array}$ & -- & $\begin{array}{c}\text { Go to Modified } \\
\text { (MT) }\end{array}$ \\
\hline
\end{tabular}

(d) L2 state transition table

Fig. 2. Example state transitions for MESI.

transition tables shown in Figure 2(c) and (d). Ack_all is triggered for the last incoming Ack and Data_Ack_all is triggered if L2's data response (which includes the Ack count) is the last message to be received.

This example illustrates the need for transient states and the additional complexities introduced by them in the MESI protocol. The larger the number of transient states, the more complex the protocol becomes. In the GEMS implementation of the MESI protocol, there are 7 transient states in $L 1$ and 14 transient states in $L 2$. Optimizations to the described baseline protocol usually incur additional transient states.

\subsection{DeNovo}

One of the main difficulties in writing and debugging parallel programs is data races. These software data races are also responsible for races in hardware with conventional hardware cache coherence protocols. These hardware races are directly responsible for most transient states in hardware protocols, and they form the root cause of much of the complexity in such protocols.

There has been a lot of software work on disciplined shared-memory programming models [Anderson et al. 2008; Gustafsson 2009; Berger et al. 2009; Blumofe et al. 1995; Budimlic et al. 2009; Ghuloum et al. 2007; Bocchino et al. 2011, 2009; Olszewski et al. 2009; Kulkarni et al. 2007; Allen et al. 2009] to eliminate or alleviate the impact of data races, with the goal of providing better programmability, debuggability, and concurrency safety of parallel programs. Along with addressing the data race problem, much of this work also provides strong safety properties, such as determinism and various disciplined forms of nondeterminism, to simplify parallel programming. DeNovo is a hardware architecture that exploits the properties of such disciplined software 
programming models to additionally simplify hardware while also providing performance, power, and scalability benefits [Choi et al. 2011].

2.2.1. Software Model. DeNovo currently uses Deterministic Parallel Java (DPJ) [Bocchino et al. 2011, 2009] as an example of a disciplined programming language to drive its design. DPJ is an extension to Java that provides properties like structured parallel control, data-race-freedom, and explicit specification of the effects of shared memory accesses. In a DPJ program, the programmer partitions the heap into named "regions" and annotates methods with summaries of read and write operations on these regions ("effects"). The DPJ compiler uses this region and effect information to guarantee determinism.

This article does not quantify software complexity; however, our software philosophy and DPJ are motivated entirely by the goal of reducing software complexity. Even today, the C++ [Boehm and Adve 2008] and Java [Manson et al. 2005] memory models do not provide any reasonable semantics for data races; therefore, a data race in these programs is a bug and imposes significant verification complexity. In contrast, DPJ provides strong safety guarantees of data-race-freedom and determinism-by-default. Programmers can reason about deterministic programs as if they were sequential. There is certainly an additional up-front burden of writing region and effect annotations in DPJ; however, arguably, this burden is mitigated by the lower debugging and testing time afforded by deterministic-by-default semantics. There is also ongoing work on partly automating the insertion of these annotations [Vakilian et al. 2009]. Overall, there is a growing part of the community that is working toward similar goals of more disciplined programming practices [WoDET 2013].

In this article, we focus on the baseline DeNovo protocol [Choi et al. 2011] targeted to deterministic codes based on the original DPJ language proposed in Bocchino et al. [2009]. DPJ and DeNovo are ongoing projects that continue to incorporate support for increasingly wider classes of programs [Bocchino et al. 2011; Bocchino and Adve 2011; Heumann et al. 2013; Sung et al. 2013, 2014]. We discuss this ongoing work and implications for general codes in Section 5.

2.2.2. DeNovo Hardware. DeNovo exploits disciplined language features for a more efficient, scalable, and simpler hardware. Specifically, structured parallel control and explicit read and write effect information enable caches to self-invalidate stale data (communicated through a compiler-inserted self-invalidate instruction for each region with write effects) and thus remove the need to maintain sharer lists and to send invalidation and acknowledgment messages. In addition, the data-race-freedom guarantee makes it possible to completely eliminate transient states from the cache coherence protocol and thus greatly reduce its complexity. As shown in [Choi et al. 2011], these benefits do not compromise the performance of the DeNovo protocol; instead, they provide opportunities for improved performance and energy without increasing complexity.

The DeNovo protocol has three states at L1, Registered (locally modified), Valid, and Invalid. L2 has the same states, too, with obvious meaning. The handling of read requests is straightforward. On a write, if the line is not registered, $L 1$ issues a registration request to $L 2$ and transitions to Registered state immediately because there are no races in the program. Similar to MESI, DeNovo also has a dirty bit at L2, which is set to indicate dirty data. The complete state transition table and a detailed description of the DeNovo protocol can be found in Choi et al. [2011].

Let us revisit the code segment from Figure 2. Figure 3(a) shows the changes to the code required to prove data-race-freedom. Specifically, the shared variable $A$ is placed in a region $R_{A}$, both the parallel phases are annotated with read and write effect summaries, and, finally, a self-invalidation instruction is inserted at the end of the second phase. 


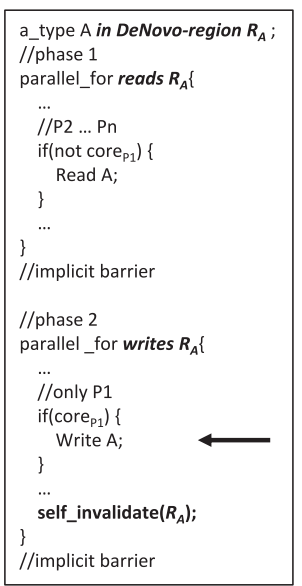

(a) Example parallel code with region and effect information

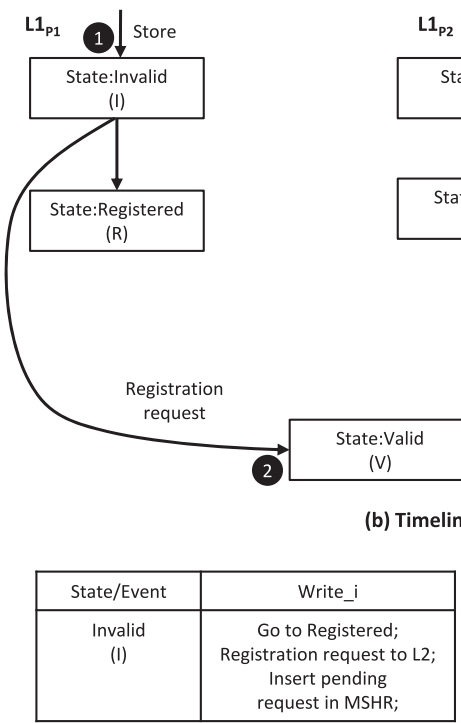

(c) L1 state transition table
$\mathrm{L1}_{\mathrm{P2}} \quad \mathrm{L}_{\mathrm{Pn}}$

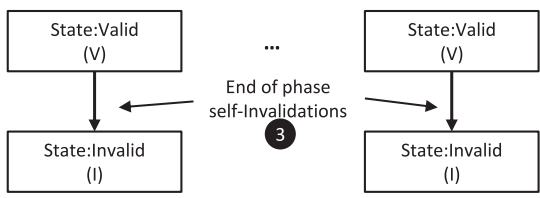

State:Registered (R)

L2/Directory

Fig. 3. Example state transitions for DeNovo.

Figure 3(b) shows the timeline of the state transitions for the DeNovo protocol and the state transition tables for the states encountered in this example are shown in Figure 3(c) and (d). Focusing again on the write instruction in the second phase, $L 1_{P 1}$ transitions directly to the Registered state without transitioning to any transient state and sends a registration request to $L 2$. $L 2$, on receiving the registration request, transitions to the Registered state. We do not show the registration response message from L2 here because it is not in the critical path and is handled by the request buffer at L1. As explained in Choi et al. [2011], the functionality of the request buffer is similar to that of a uniprocessor system and is invisible to the outside world. At the end of the phase, each core executes a self-invalidate instruction on region $R_{A}$. This instruction triggers the invalidation of all the data in region $R_{A}$ in the $L 1$ cache of its core (except for data in Registered state since this data is known to be up-to-date).

As an optimization, the $L 1$ maintains an additional touched bit for each word. This bit is set on a read, indicating that this data will stay up-to-date until the end of the phase. This is guaranteed by data-race-freedom because there cannot be concurrent writers to the same location. The self-invalidation operation now skips invalidating the data that is both Valid and touched. These touched bits are reset at the end of the parallel phase.

This example illustrates how the absence of transient states makes the DeNovo protocol simpler than MESI.

\section{MODELING FOR PROTOCOL VERIFICATION}

We use the Mur $\varphi$ model checking tool [Dill et al. 1992; Ip and Dill 1993, 1996] to verify the simple word-based protocols (equal address, communication and coherence granularity as explained in Choi et al. [2011]) of DeNovo and MESI. We derived the MESI model from the GEMS implementation [Martin et al. 2005]. We derived the DeNovo model from our implementation. To keep the number of states explored (by $\operatorname{Mur} \varphi$ ) tractable, as is common practice, we used a single address, single region (only for DeNovo), two data values, and two cores. We modeled private $L 1$ caches, a unified 


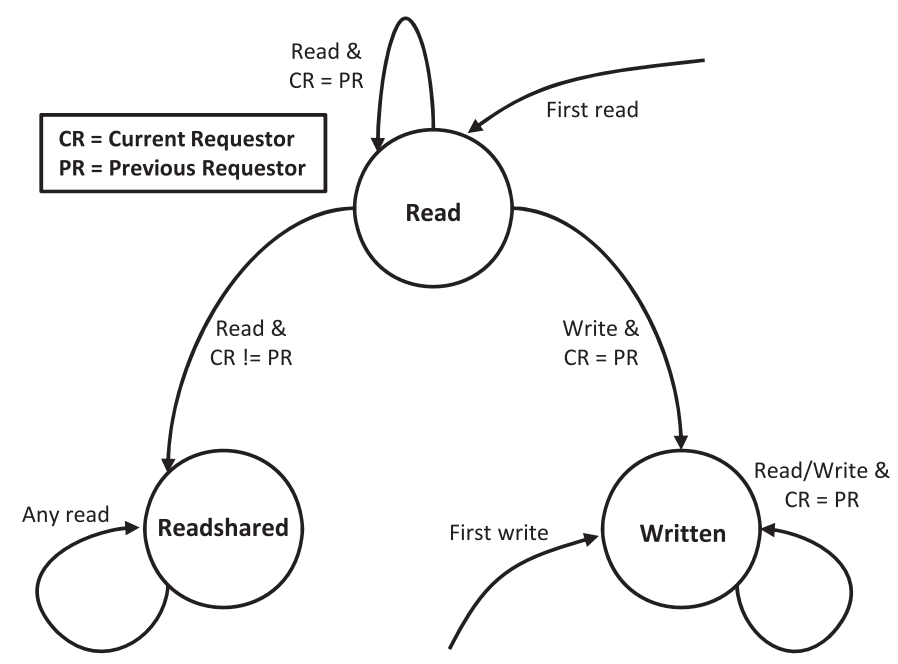

Fig. 4. State transitions for AccessStatus data structure in a given phase. An access for which there is no transition cannot occur and is dropped.

L2, an in-cache directory (for MESI), and an unordered full network with separate request and reply links. Both models allow only one request per $L 1$ in the rest of the memory hierarchy. Because we modeled only one address, we modeled replacements as unconditional events that can be triggered at any time. To enable interactions across multiple parallel phases (cross-phase) in both the models, we introduced the notion of a phase boundary by modeling it as a sense-reversing barrier. Finally, we modeled the data-race-free guarantee for DeNovo by limiting conflicting accesses. We explain each of these attributes in detail in the following sections.

\subsection{Abstract Model}

To reduce the amount of time and memory used in verification, we modeled the processors, addresses, data values, and regions as scalarsets [Ip and Dill 1996], a datatype in $\operatorname{Mur} \varphi$ that takes advantage of the symmetry in these entitites while exploring the reachable states. A processor is modeled as an array of cache entries consisting of $L 1$ state information along with protocol-specific fields like the region field and the touched bit for DeNovo. L1 state is one of three possible states for DeNovo or one of 11 possible states for MESI. Similarly, L2 is also modeled as an array of cache entries, each with L2 state information, a dirty bit, and other protocol-specific details like sharer lists for MESI. L2 state is one of three possible states for DeNovo or one of 18 possible states for MESI. Memory is modeled as an array of addresses storing data values.

3.1.1. Data-Race-Free Guarantee for DeNovo. To model the data-race-free guarantee from software for DeNovo, we used an additional data structure called AccessStatus. As shown in Figure 4, this data structure maintains the current status (read, readshared, or written) and the core id of the last requestor for every address in the model. The current status and the last requestor determine the reads and writes that cannot occur in a data-race-free program and are thus disallowed in the model.

On any read, if it is the first access to this address in this phase, then status is set to read. If status is already set to read and the requesting core is not the same as the last requestor, then status is set to readshared. If status is readshared, then it stays the same on the read. If status is written and the requesting core is the same as the last requestor, it stays as written. On the other hand, if the requesting core is not the same 
as the last requestor, then this access is not generated in the model since it violates the data-race-freedom guarantee.

Similarly, on any write, if it is the first access to this address or if the requesting core is the same as the last requestor, then status is set to write. If status is either readshared or the requesting core is not the same as the last requestor, then this access is not generated to adhere to the data-race-free guarantee.

The AccessStatus data structure is reset for all the addresses at the end of a phase.

3.1.2. Cross-phase Interactions. We modeled the end of a parallel phase (and the start of the next phase) using a sense-reversing barrier implementation [Mellor-Crummey and Scott 1991]. This event (end-of-phase) can be triggered at any time; that is, with no condition. The occurrence of end-of-phase is captured by a flag, releaseflag. This event occurs per core and stalls the core from issuing any more memory requests until (1) all the pending requests of this core are completed (i.e., the $L 1$ request buffer is empty), and (2) all other cores reach the barrier. The completion of end-of-phase is indicated by resetting the releaseflag flag. Figure 5 shows the Mur $\varphi$ code for end-ofphase implementation for the DeNovo protocol. The spinwaiting flag indicates that the current core is waiting for other cores to reach the barrier. When a core enters the barrier for the first time, the local sense of the barrier (localsense) is reversed, thus indicating entering a new barrier; barrier count (barcount) is updated; and the spinwaiting flag is set. If it is the last one to enter the barrier, the core also notifies all other cores about the end of barrier by assigning barrier its localsense. It also resets the barcount and releaseflag. Once a core reaches the barrier, we also modeled self-invalidations and unsetting touched bits for DeNovo. The code for MESI is similar except for DeNovo-specific operations like self-invalidation and unsetting touched bits.

\subsection{Invariants}

This section discusses the invariants we checked to verify the MESI and DeNovo protocols. The MESI invariants are based on prior work in verification of cache coherence protocols [Dill et al. 1992; McMillan and J. 1991]. The DeNovo invariants are analogous as further described later. (Adding more invariants does not affect the verification time appreciably because the number of system states explored is still the same.)

3.2.1. MESI Invariants. We used five invariants to verify the MESI protocol [Dill et al. 1992; McMillan and Schwalbe 1991].

-Empty sharer list in Invalid state. This invariant asserts that the sharer list is empty when $L 2$ transitions to Invalid state, and it ensures that there are no L1s sharing the line after $L 2$ replaces the line.

-Empty sharer list in Modified state. This invariant asserts that the sharer list is empty when L2 transitions to Modified state.

-Only one modifiable or exclusive cache copy. This invariant checks that there is only one cache copy in either Modified or Exclusive state. It is also a violation for a cache line to be in both these states at the same time.

-Data value consistency at $L 1$. When $L 1$ is in Shared state and $L 2$ is also in Shared state, the data values should be the same at both $L 1$ and $L 2$. Indirectly, this invariant also makes sure that all the $L 1$ s have the same data value when in Shared state.

-Data value consistency at L2. This invariant checks that when $L 2$ is in Shared state and dirty bit is not set, L2's data value should be the same as at memory.

3.2.2. DeNovo Invariants. We modeled six invariants for the DeNovo protocol. Because there is no sharer list maintained in the DeNovo protocol, we do not check for the first two invariants of the MESI protocol. The first three invariants of the DeNovo protocol 


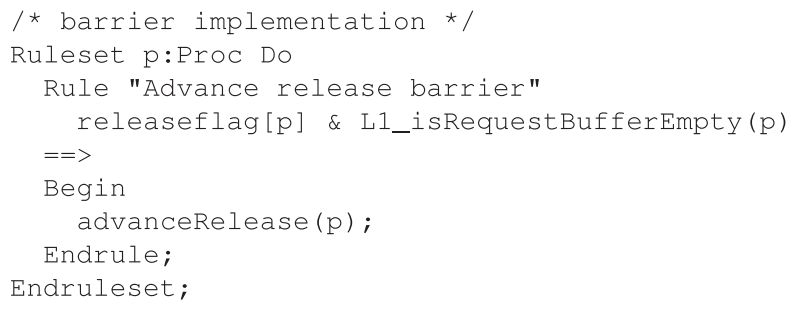

(a) $\operatorname{Mur} \varphi$ barrier rule.

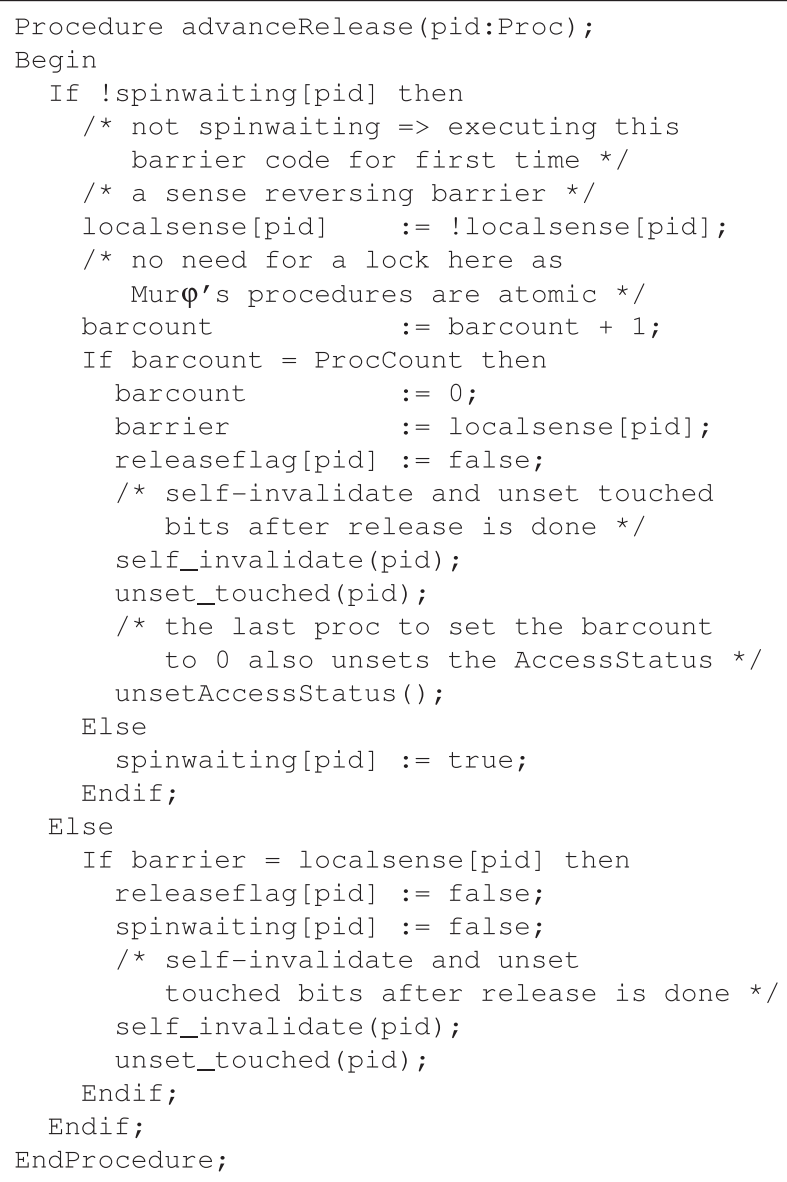

(b) Mur $\varphi$ procedure for implementing a sense-reversing barrier.

Fig. 5. Mur $\varphi$ code for end-of-phase implementation for DeNovo as a sense-reversing barrier. (a) Rule that gets triggered when inside the barrier indicated by releaseflag and empty $L 1$ request buffer (no outstanding requests) and (b) implementation of the sense-reversing barrier, including calls to end-of-phase operations like self-invalidation instruction and unsetting of touched bits. 
are similar to the last three invariants of the MESI protocol. The last three invariants of the DeNovo protocol are checks on the touched bit functionality.

-Only one modifiable cache copy. There cannot be two modifiable $L 1$ cache copies in the system at the same time. This invariant checks that there are never two $L 1$ caches in Registered state for the same line at the same time.

-Data value consistency at L1. This invariant has two parts: (1) If L1 is in Valid state and touched bit is set (value is read in this phase) and L2 is also in Valid state, then the data values should be the same at both $L 1$ and $L 2$. (2) If $L 1$ is in Valid state and touched bit is set and some other $L 1$ is in Registered state, ${ }^{4}$ the data values should match.

-Data value consistency at L2. This invariant checks that when L2 is in Valid state and dirty bit is not set, L2's data value should be the same as at memory.

- Touched bit on a write. On a write, this invariant checks that no other cache has the touched bit set to true. This verifies that the touched bit is implemented correctly.

- Touched bit on a read. Similar to the above, on a read, this invariant checks that the only cache lines that can have the touched bit set to true (for cores other than the requestor) are the ones in Valid state.

-Unsetting touched bits. Finally, this invariant checks that all the touched bits are set to false at the end of the phase.

\section{RESULTS}

Through model checking, we found six bugs (including two deadlock scenarios) in MESI and three bugs in DeNovo. ${ }^{5}$ A deadlock occurs when all the entities in the system (all $L 1$ s and $L 2$ ) stop making any forward progress. Mur $\varphi$ checks for deadlock by default. In MESI, all the bugs except one of the deadlocks were caused by protocol races between $L 1$ writebacks and other cache events. The other deadlock was caused by incorrect handling of clean replacement at the $L 2$.

Most of these bugs found in MESI involved subtle data races and took several days to track, debug, and fix. All three bugs found in DeNovo were simple to fix and showed mistakes in translating our internal high level specification into the implementation (i.e., their solutions were already present in our internal high level description of the protocol).

Each of the bugs found in MESI and DeNovo is described in detail next. In all these descriptions, we consider a single address. $L 1_{P 1}, L 1_{P 2}$, and $L 2$ indicate the cache lines corresponding to this address in core $P 1$, core $P 2$, and $L 2$, respectively. As mentioned in Section 2 , we assume an in-cache directory at $L 2$, and hence we use the words directory and $L 2$ interchangeably.

\subsection{MESI Bugs}

We first discuss the six bugs found in the MESI protocol. We list them in decreasing order of complexity and the amount of change to the code required to fix them. ${ }^{6}$

Bug 1. The first bug is caused by a race between an $L 1$ writeback and a write request by some other $L 1$. Figure 6 shows the events that lead to this bug. Let us assume that initially $L 1_{P 1}$ is in Modified state, $L 1_{P 2}$ is in Invalid state, and $L 2$ records that the

\footnotetext{
${ }^{4}$ This is possible in DeNovo because registration at the other $L 1$ may have happened in a previous parallel phase.

${ }^{5}$ We note that these bugs were discovered in 2011 when DeNovo was (and still is) much less mature than the GEMS MESI protocol. The latter was released in 2007 and had been used by many researchers over the course of four years when we found the bugs.

${ }^{6}$ We confirmed the fixes for the MESI bugs in a personal email exchange with Arkaprava Basu, one of the developers of the GEMS simulation suite.
} 


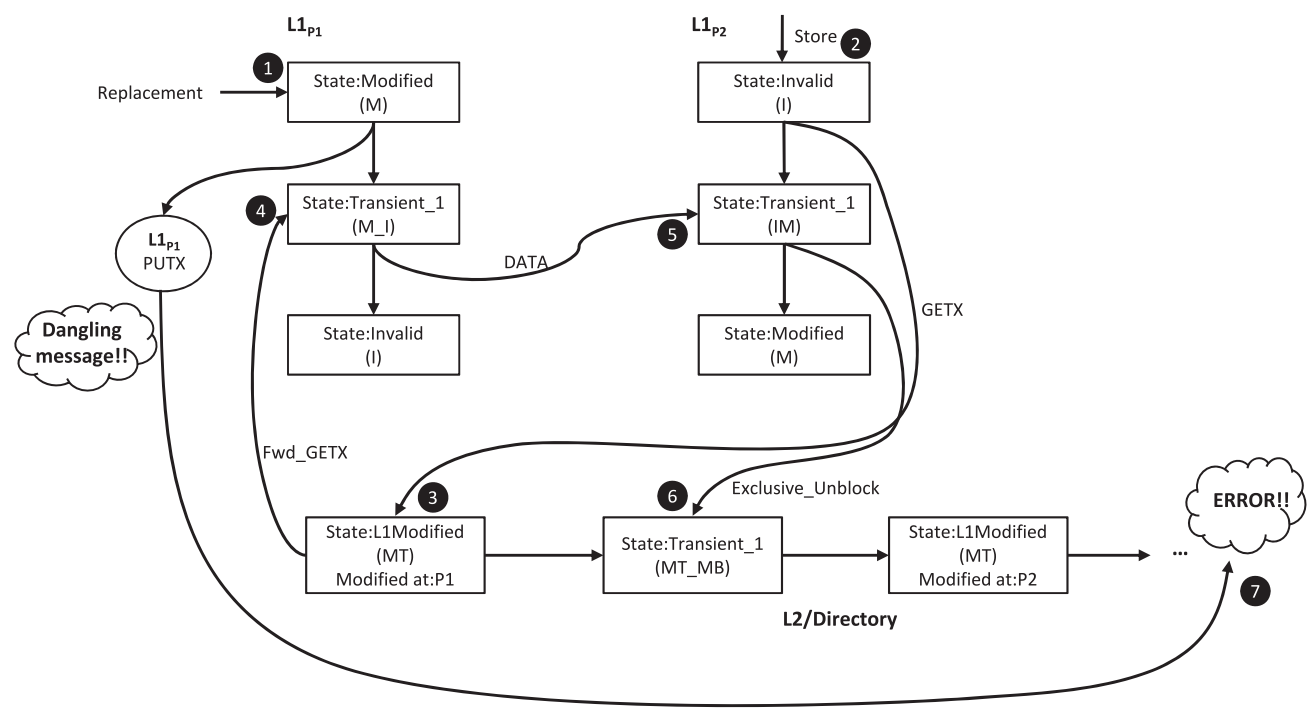

Fig. 6. MESI Bug 1 showing a race between an $L 1$ writeback and a remote write request.

cache entry is modified in $L 1_{P 1}$. Then $L 1_{P 1}$ issues a replacement (event 1 in Figure 6) triggering a writeback (PUTX) and transitions to a transient state waiting for an acknowledgment to this writeback request. Meanwhile, $L 1_{P 2}$ issues a write request (event 2) triggering GETX to $L 2$. $L 2$ first receives $G E T X$ from $L 1_{P 2}$ (event 3). It forwards the request to $L 1_{P 1}$ and waits for an acknowledgment from $L 1_{P 2} . L 1_{P 1}$, on receiving the GETX request (event 4), forwards the data to $L 1_{P 2}$ and transitions to Invalid state. Then $L 1_{P 2}$, on receiving the data from $L 1_{P 1}$ (event 5) transitions to Modified state and unblocks the directory, which in turn records that the cache entry is now modified in $L 1_{P 2}$. But the writeback $(P U T X)$ sent by $L 1_{P 1}$ is still in the network and it can reach the directory at any time because we have an unordered network (event 7), causing an error. For example, suppose $L 1_{P 1}$ later services a write request invalidating $L 1_{P 2}$ and the directory is appropriately updated (not shown in the figure). $L 1_{P 1}$ 's writeback $(P U T X)$ then reaches the directory, which is clearly an error. The bug was found when the writeback acknowledgment from $L 2$ reached $L 1_{P 1}$, triggering a "missing transition" failure ( $L 1_{P 1}$ does not expect a writeback acknowledgment in Modified state).

We solved this problem by not transitioning $L 1_{P 1}$ to Invalid state on receiving $L 1_{P 2}$ 's GETX request. $L 1_{P 1}$ now sends $D A T A$ to $L 1_{P 2}$ as before, but continues to stay in the transient state, M_I. The write request from $L 1_{P 1}$, which triggered the bug in the previous example, is now kept pending because $L 1_{P 1}$ is in a transient state. We also added a transition at the $L 2$ to send a writeback acknowledgment when the requester is not the owner in the directory's record. $L 1_{P 1}$ transitions to Invalid state on receiving the writeback acknowledgment from $L 2$. With this, there is no longer a dangling PUTX in the network and the problem is solved. The trace for this bug involved multiple writes to the same memory location in a parallel phase. This scenario does not arise in DeNovo because the software guarantees data-race-freedom.

Bug 2. The second bug is similar to the first except that it is caused by a race between an $L 1$ writeback and a read request by some other $L 1$.

The first two bugs were the most complex to understand and fix. Most of the time was spent in discovering the root cause of the bugs and developing a solution in an 
already complex protocol. The solutions to these bugs required adding two new cache events and eight new transitions to the protocol.

Bug 3. The third bug is caused by an unhandled protocol race between $L 2$ and $L 1$ replacements. To begin with, $L 1_{P 1}$ is in Exclusive state and $L 2$ records that $P 1$ is the exclusive owner. Then, both $L 2$ and $L 1$ replace the lines simultaneously, triggering invalidation and writeback messages, respectively. $L 1_{P 1}$, on receiving the invalidation message, transitions to Invalid state and sends its data to $L 2$. On receiving this data, $L 2$ completes the rest of the steps for the replacement. In the end, both $L 1$ and $L 2$ have transitioned to Invalid states, but the initial writeback message from $L 1$ is still in the network and this is incorrect. The bug was found when the writeback acknowledgment (issued by $L 2$ on receiving the dangling writeback message) reaches $L 1_{P 1}$ when it is not expecting one and hence triggers a "missing transition" error.

This bug can be fixed by not sending the data when $L 1$ receives an invalidation message and by treating the invalidation message itself as the acknowledgment for $L 1$ 's earlier writeback message. Also, the $L 1$ writeback message is treated as the data response for the invalidation message at $L 2$. The fix required adding four new transitions to the protocol.

Bug 4. The fourth bug results in a deadlock situation. It is caused by an incorrectly handled protocol race between an Exclusive_unblock (response sent to unblock L2 on receiving an exclusive access) and an $L 1$ writeback issued by the same $L 1$ (issued after sending Exclusive_unblock). Initially, L2 is waiting for an Exclusive_unblock in a transient state transitioned from Invalid state. In this transient state, when $L 2$ receives an $L 1$ writeback, it checks whether this writeback came from the current owner or not. The owner information is updated at $L 2$ on receiving the Exclusive_unblock message. Here, $L 1$ writeback (racing with Exclusive_unblock from the same $L 1$ ) reached $L 2$ first and $L 2$ incorrectly discarded the $L 1$ writeback because the owner information at $L 2$ did not match the sender of the $L 1$ writeback. This incorrect discarding of the $L 1$ writeback results in a deadlock.

This bug can be fixed by holding the $L 1$ writeback to be serviced until Exclusive _ unblock is received by $L 2$. This requires adding a new transition and additional buffering to hold the stalled request to the protocol.

Bug 5. The fifth bug is similar to the fourth (race between Exclusive_unblock and L1 writeback), but instead $L 2$ is initially in Shared state. The fix for this bug required adding two new transitions and additional buffering to hold the stalled requests to the protocol.

Bug 6. The last bug results in a deadlock scenario due to an incorrect transition by $L 2$ on a clean replacement. It transitions to a transient state awaiting an acknowledgment from memory even though the transition did not trigger any writeback. The fix was simple and required transitioning to Invalid state instead.

\subsection{DeNovo Bugs}

We next discuss the three bugs found in the DeNovo protocol. The first bug is a performance bug and the last two are correctness bugs, both of which are caused by races related to writebacks.

Bug 1. The first of the three bugs found was caused by not unsetting the dirty bit on replacement of a dirty $L 2$ cache line. Assume that L2 is initially in Valid state and the dirty bit is set to true. Then, on $L 2$ replacement, it transitions to Invalid state and writes back data to memory. But the dirty bit is mistakenly not unset. This bug was found when Mur $\varphi$ tried to replace the line in Invalid state as the dirty bit was 
set to true (the model triggers a replacement event by only checking the dirty bit). The model, legitimately, did not have an action specified for a replacement event in the Invalid state, thus resulting in a "missing transition" error. However, the actual implementation did have an action (incorrectly) that triggered unnecessary writebacks to memory, which should be silent replacements instead. This turned out to be a rare case to hit in the simulation runs.

Bug 2. This occurs because an $L 2$ initiated writeback and future requests to the same cache line are not serialized. Initially, $L 1_{P 1}$ is in Registered state and $L 2$ knows $P 1$ as the registrant. On replacing the line, $L 2$ sends a writeback request to $L 1$. $L 1$ replies to this writeback request by sending the data to $L 2$ and transitions to Valid state. ${ }^{7}$ Then, on receiving the writeback from $L 1, L 2$ sends an acknowledgment to $L 1$ and, in parallel, sends a writeback to memory and waits for an acknowledgment. Meanwhile, let us assume that $L 1$ issued a registration request (on receiving a store request) and successfully registers itself with $L 2$. At this point, yet another $L 2$ replacement was triggered, finally leading to multiple writebacks to memory in flight. This is incorrect because the writebacks can be serviced out of order. $\operatorname{Mur} \varphi$ found this bug when an assertion failed inside the implementation of $L 2$ 's request buffer.

The real source of this bug is allowing $L 1$ registration to be serviced at $L 2$ while a writeback to memory is pending. The fix involves serializing requests to the same location at $L 2$-in this case, the $L 1$ registration request behind the writeback to memory. This was already present in our high level specification but was missed in the actual protocol implementation. It did not involve adding any new states or transitions to the protocol.

Bug 3. The last bug is due to a protocol race where both the $L 1$ s and the $L 2$ replace the line. This bug involves both cores and cross-phase interactions. The events that lead to the bug are shown in Figure 7. At the beginning of the phase, let us assume that $L 1_{P 1}$ is in Invalid state and $L 1_{P 2}$ is in Registered state (from the previous phase). $L 1_{P 2}$ replaces the line (event 1 in Figure 7 ) and issues a writeback (PUTX) to $L 2$. While this writeback is in flight, $L 1_{P 1}$ successfully registers itself with $L 2$ (events $2-4$ ) ( $L 2$ redirects the request to $L 1_{P 2}$ because it is the current registrant). This is followed by a replacement by $L 1_{P 1}$ (event 5 ), thus triggering another writeback (PUTX) to L2. $L 2$ first receives the writeback from $L 1_{P 1}$ (event 6) and responds by sending an acknowledgment and transitioning to Valid state while setting the dirty bit to true. Now, L2 also replaces the line (event 7) transitioning to Invalid state and issues a writeback to memory. But the writeback from $L 1_{P 2}$ is still in flight. This writeback now reaches $L 2$ (event 8) while in Invalid state (because we model an unordered network). The implementation did not handle this case and resulted in a "missing transition" failure. This bug can be easily fixed by adding a transition to send an acknowledgment to $L 1_{P 2}$ 's writeback without the need for triggering any actions at $L 2$.

\subsection{Analysis}

The bugs just described for both MESI and DeNovo show that cache line replacements and writebacks, when interacting with other cache events, cause subtle races and add to the complexity of cache coherence protocols. Fixes to bugs in the MESI protocol needed adding new events and several new transitions. On the other hand, fixing bugs in the DeNovo protocol was relatively easy since it lacks transient states even for races related to writebacks.

\footnotetext{
${ }^{7}$ In DeNovo, the L2 cache is inclusive of only the registered lines in any L1. Hence, it is possible for $L 1$ to transition from Registered to Valid on receiving a writeback request from $L 2$.
} 


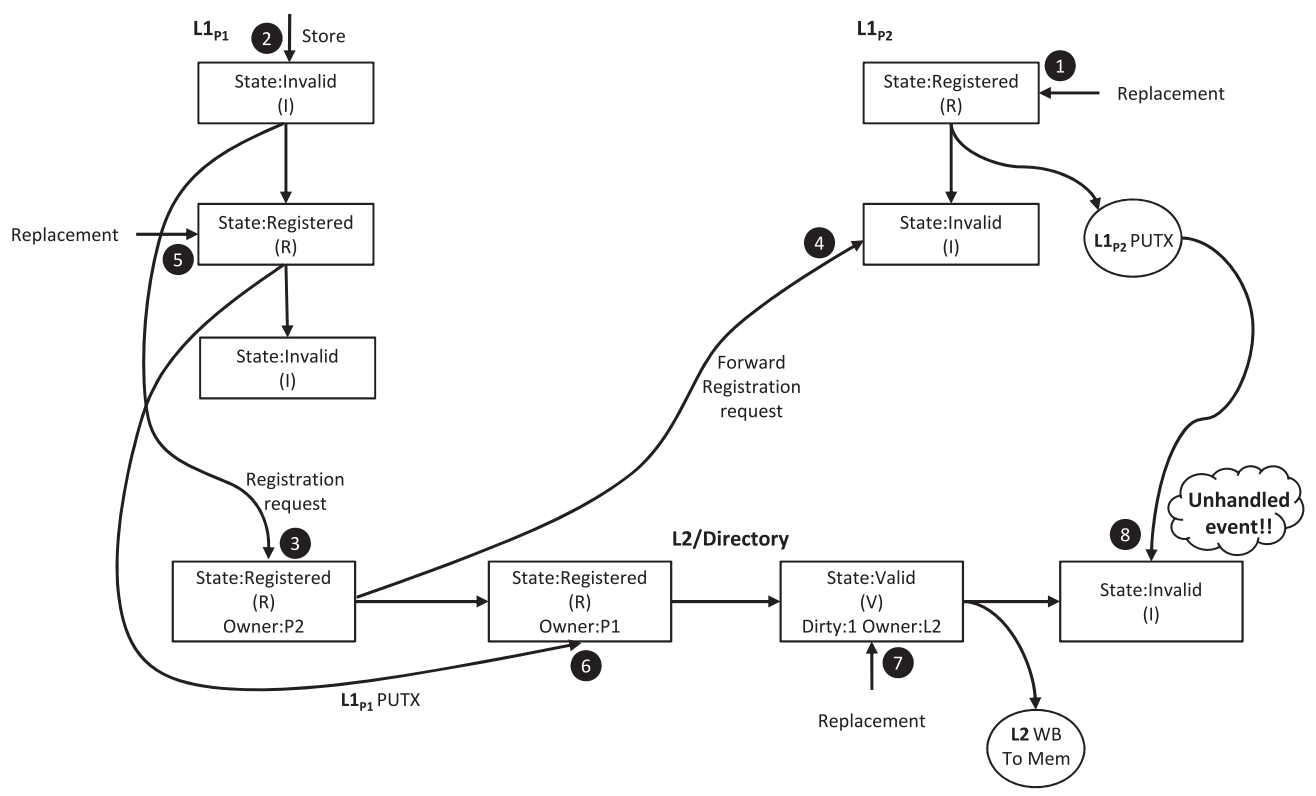

Fig. 7. DeNovo Bug 3 showing a race between replacements at both the L1s and the L2. This figure doesn't show the request buffer entries for L2 and for writeback entries at L1.

\subsection{Verification Time}

After fixing all the bugs, we ran the models for both MESI and DeNovo on Mur $\varphi$ as described in Section 3. The model for MESI explores 1,257,500 states in 173 seconds, whereas the model for DeNovo explores 85,012 states in 8.66 seconds. These are the number of distinct system states exhaustively explored by the model checking tool. The state space and runtime both grow significantly when we increase the parameters in the verification model. For example, when we modeled two addresses, we were able to finish running DeNovo without any bugs being reported, but we ran out of system memory (32GB) for MESI. This indicates (1) the simplicity and reduced verification overhead for DeNovo compared to MESI, and (2) the need for more scalable tools amenable to non-expert to deal with more conventional hardware coherence protocols in a more comprehensive way.

\section{RELATED WORK}

Hardware cache coherence protocols are inherently complex. There is an ongoing debate about whether we have tamed the complexity of hardware coherence protocols or whether we should abandon them and replace them with their software counterparts. While some predict that hardware cache coherence is here to stay [Martin et al. 2012] and continue to extend such protocols in newer, more complex ways [Aisopos and Peh 2011; Zhao et al. 2013], others propose and build systems with software-directed coherence [Borkar 2011; Choi et al. 2011; Howard et al. 2010; IntelSCC 2009; Kelm et al. 2009]. Our work offers an objective case study to show that hardware coherence remains quite complex and that a hardware-software co-design approach can provide a simpler alternative. This section first discusses several existing techniques to verify hardware coherence protocols (Section 5.1). Next, we discuss related work on redesigning conventional hardware coherence protocols specifically to address verification complexity (Section 5.2). 


\subsection{Verification Techniques}

Hardware coherence protocols have numerous transient states and hard-to-cover race conditions making it very difficult to find all the bugs using just simulations or random testing. Hence, formal methods like model checking are often employed to verify their correctness. Model checking is a technique to verify the properties of a system by exhaustive exploration of the state space [Clarke and Emerson 1982; Queille and Sifakis 1982]. McMillan and Schwalbe's seminal work on model checking the Encore Gigamax protocol [McMillan and Schwalbe 1991] was the first to apply model checking to verify cache coherence protocols.

Complex systems often exhibit a lot of regularity and symmetry. Ip and Dill developed $\operatorname{Mur} \varphi$ [Dill et al. 1992; Ip and Dill 1993, 1996], which exploits these characteristics by grouping together similar states to verify a reduced state graph instead of the full one. This helps to greatly reduce the amount of time and memory used in verification. Mur $\varphi$ is a widely used tool to formally verify cache coherence protocols; for example, Sun RMO memory model [Park and Dill 1995], Sun S3.mp multiprocessor [Pong et al. 1998], Cray SV2 protocol [Abts et al. 2000], and Token coherence protocol [Burckhardt et al. 2005].

In this article, we use $\operatorname{Mur} \varphi$ for our protocol verification work. Using model checking tools like Mur $\varphi$ for verifying cache coherence protocols is not new per se. This article, nevertheless, does discuss some extensions to the canonical coherence protocol modeling technique to model the guarantees provided by disciplined programming languages (namely, data-race-freedom and a disciplined parallel phase behavior). The main contribution of this work is to provide a detailed experience of the verification process of a state-of-the-art, publicly available, mature, modern hardware coherence protocol implementation (MESI). We further compare this experience with that of verifying a protocol driven by a hardware-software co-design approach (DeNovo), motivated by simplifying both software and hardware through the same mechanisms (while providing opportunities for performance and energy improvements).

Explicit state exploration model checking tools traditionally have the problem of state space explosion limiting the scalability of such tools. As mentioned in Section 4.4, we ran out of system memory when we increased the verification parameters. We tried a distributed model checker based on $\operatorname{Mur} \varphi$, Preach [Bingham et al. 2010] (similar to Eddy Murphi [Melatti et al. 2009]). But PReach did not help us reduce the number of states explored, and hence the memory footprint stayed the same.

There are other verification techniques that do not have this state explosion problem and can scale to larger systems. Parametric verification [Chou et al. 2004; McMillan 2001; O'Leary et al. 2009] and theorem proving [Seungjoon and David 1996] are two such techniques. Several of these techniques are sometimes combined together to verify a given system. For example, the verification of Token Coherence [Burckhardt et al. 2005] is achieved by combining assume-guarantee reasoning and structural induction (in addition to model checking). Even though the techniques employed were able to verify the protocol, in practice, these techniques are either hard for nonspecialists to use or error-prone because of laborious manual intervention [Zhang et al. 2014]. There have been proposals to automate parametric verification techniques and minimize manual intervention [Chou et al. 2004], but such techniques impose severe limitations on the protocols that can be verified. DeNovo, in contrast, is a simpler protocol and makes it feasible to verify with easy-to-use verification techniques, such as an explicit state model checker. A general survey of various techniques used to verify cache coherence protocols can be found in Pong and Dubois [1997].

\subsection{Redesigning Hardware Coherence Protocols}

There has been some work on redesigning the hardware cache coherence protocol itself to address the verification problem. A recent proposal, PVCoherence [Zhang 
et al. 2014], lists various guidelines for designing cache coherence protocols so that they can be verified using existing automatic parametric verification techniques. The verification technique used in the paper, Simple-PV, uses an automatic tool to generate the parametric model and then Mur $\varphi$ to verify the generated model. When the proposed guidelines were applied to the MOESI protocol to make it amenable to parametric verification (in the process also making it even more complex), the authors noticed that the resulting protocol could not be verified by $\operatorname{Mur} \varphi$ and needed even more changes. The final protocol was verifiable but showed performance degradation (e.g., average $5 \%$ and up to $13.8 \%$ increase in network traffic). In Fractal Coherence [Zhang et al. 2010; Voskuilen and Vijaykumar 2014], the verification for arbitrary number of cores is made possible by just verifying the minimum system for correctness and verifying that the whole system has fractal behavior. But Fractal Coherence requires addition of states and messages to an existing cache coherence protocol (MOSI, as described in Zhang et al. [2010]) to maintain fractal behavior. Thus, it enables verification of the entire system at the expense of increasing the complexity of the base protocol. Also, a specific implementation of Fractal Coherence, TreeFractal, shows a performance degradation $(>10 \%)$ when compared to traditional protocols. FlatFractal [Voskuilen and Vijaykumar 2014] proposes techniques to eliminate some of the limitations of TreeFractal, making FlatFractal's performance comparable to the base implementation on 16 cores. But when scaled to 32 cores, FlatFractal shows up to $40 \%$ degradation in total execution time. Atomic Coherence [Vantrease et al. 2011] attempts to simplify the coherence protocol by separating out the races from the protocol. The design avoids the protocol races by requiring each coherence action to be guarded by a mutex. It uses nanophotonics for performing these mutex operations with low latency because they are now on the critical path. As a result, Atomic Coherence eliminates transient states in the coherence protocol but heavily relies on a specific type of on-chip network.

In contrast to these approaches, DeNovo reduces the complexity of the cache coherence protocol by following a hardware-software co-design approach that eliminates races and thereby reduces the overall verification overhead. The basic DeNovo protocol is shown to be performance-competitive with MESI, and its simplicity enables optimizations that significantly improve performance and network traffic, thereby also potentially saving energy. The DeNovo coherence protocol used in this article assumes deterministic software. DeNovo is an ongoing research project seeking to broaden the class of software supported by such simple protocols. Recently, we extended DeNovo to support disciplined lock-based programs using dynamically generated access signatures to guide self-invalidations [Bocchino et al. 2011; Sung et al. 2013, 2014]. These extensions are rather small, and we believe can be verified independently. We are also developing simple extensions to support more complex lock-free synchronization algorithms. With these extensions, DeNovo will be able to support all codes correctly as long as synchronization operations are explicit. ${ }^{8}$ For legacy codes that do not contain annotations for self-invalidations or generating dynamic access signatures, performance (but not correctness) may be affected. DeNovo's vision of using disciplined parallel programming practices for better performance and programmability is shared by other projects as well, including for complex system code such as operating systems (e.g., Barrelfish [Baumann et al. 2009]). This paper provides an objective study to quantify the complexity advantages of such approaches.

\section{CONCLUSION}

With the era of manycores, there is a renewed debate in the community as to whether we have tamed the complexity of hardware coherence enough to employ it in systems

\footnotetext{
${ }^{8}$ Recent standards efforts for popular languages like Java, C, and C++ already require making synchronizations explicit.
} 
with hundreds of cores or whether we should abandon it for other software alternatives. Although some have argued that hardware coherence is here to stay [Martin et al. 2012] and continue to work on even more complex extensions [Aisopos and Peh 2011], others are proposing systems that use software-directed coherence [Borkar 2011; Choi et al. 2011; Howard et al. 2010; IntelSCC 2009; Kelm et al. 2009]. This article adds objective results from a concrete case study to this debate. We attempted to formally verify a publicly available, state-of-the-art implementation of the MESI protocol. Surprisingly, we found that after four years of extensive use in the architecture community, the protocol implementation still had several bugs. These bugs were hard to diagnose and fix, requiring new state transitions. In contrast, verifying a far less mature, hardwaresoftware co-designed protocol, DeNovo, revealed fewer bugs that were much easier to fix. After the bug fixes, we found that MESI took 20X longer to model check and required significant simplifying assumptions for the verification to be tractable. We conclude that even with a multitude of prior work, hardware coherence protocols are still difficult to design and understand. Furthermore, although it is difficult to define a single metric to quantify the relative complexity of protocols or to generalize from two design points, our results indicate that hardware-software co-designed protocols may offer a simpler alternative. This article also reinforces the necessity of employing formal verification techniques to prove the correctness of these protocols, but these techniques still require simplifying assumptions.

\section{REFERENCES}

Dennis Abts, David J. Lilja, and Steve Scott. 2000. Toward complexity-effective verification: A case study of the Cray SV2 cache coherence protocol. In Proceedings of the Workshop on Complexity-Effective Design held in conjunction with the 27th Annual International Symposium on Computer Architecture (ISCA2000).

Dennis Abts, Steve Scott, and David J. Lilja. 2003. So many states, so little time: Verifying memory coherence in the cray X1. In IPDPS.

Konstantinos Aisopos and Li-Shiuan Peh. 2011. A systematic methodology to develop resilient cache coherence protocols. In Proceedings of the 2011 44th IEEE/ACM International Symposium on Microarchitecture (MICRO'11).

Matthew D. Allen, Srinath Sridharan, and Gurindar S. Sohi. 2009. Serialization sets: A dynamic dependencebased parallel execution model. In PPoPP. 85-96. DOI : http://dx.doi.org/10.1145/1504176.1504190

Zachary Anderson, David Gay, Rob Ennals, and Eric Brewer. 2008. SharC: Checking data sharing strategies for multithreaded C. In PLDI. 149-158. DOI : http://dx.doi.org/10.1145/1375581.1375600

Andrew Baumann, Paul Barham, Pierre-Evariste Dagand, Tim Harris, Rebecca Isaacs, Simon Peter, Timothy Roscoe, Adrian Schüpbach, and Akhilesh Singhania. 2009. The multikernel: A new OS architecture for scalable multicore systems. In Proceedings of the ACM SIGOPS 22nd Symposium on Operating Systems Principles (SOSP'09). ACM, New York, NY, 29-44. DOI : http://dx.doi.org/10.1145/1629575.1629579

Emery D. Berger, Ting Yang, Tongping Liu, and Gene Novark. 2009. Grace: Safe multithreaded programming for C/C++. In OOPSLA. 81-96. DOI : http://dx.doi.org/10.1145/1640089.1640096

Brad Bingham, Jesse Bingham, Flavio M. de Paula, John Erickson, Gaurav Singh, and Mark Reitblatt. 2010. Industrial strength distributed explicit state model checking. In Proceedings of the 2010 9th International Workshop on Parallel and Distributed Methods in Verification, and 2nd International Workshop on High Performance Computational Systems Biology (PDMC-HIBI'10). IEEE Computer Society, Washington, DC, 28-36. DOI : http://dx.doi.org/10.1109/PDMC-HiBi.2010.13

Nathan Binkert, Bradford Beckmann, Gabriel Black, Steven K. Reinhardt, Ali Saidi, Arkaprava Basu, Joel Hestness, Derek R. Hower, Tushar Krishna, Somayeh Sardashti, Rathijit Sen, Korey Sewell, Muhammad Shoaib, Nilay Vaish, Mark D. Hill, and David A. Wood. 2011. The gem5 simulator. SIGARCH Computer Architecture News 39, 2 (August 2011), 1-7. DOI : http://dx.doi.org/10.1145/2024716.2024718

Robert D. Blumofe, Christopher F. Joerg, Bradley C. Kuszmaul, Charles E. Leiserson, Keith H. Randall, and Yuli Zhou. 1995. Cilk: An efficient multithreaded runtime system. In PPoPP. 207-216.

Robert L. Bocchino and Vikram S. Adve. 2011. Types, regions, and effects for safe programming with objectoriented parallel frameworks. In Proceedings of the 25th European Conference on Object-oriented Programming(ECOOP'11). Springer-Verlag, Berlin, 306-332. 
Robert L. Bocchino, Jr., Vikram S. Adve, Danny Dig, Sarita V. Adve, Stephen Heumann, Rakesh Komuravelli, Jeffrey Overbey, Patrick Simmons, Hyojin Sung, and Mohsen Vakilian. 2009. A type and effect system for deterministic parallel Java. In OOPSLA. 97-116. DOI : http://dx.doi.org/10.1145/1639949.1640097

Robert L. Bocchino, Jr., Stephen Heumann, Nima Honarmand, Sarita V. Adve, Vikram S. Adve, Adam Welc, and Tatiana Shpeisman. 2011. Safe nondeterminism in a deterministic-by-default parallel language. In Proceedings of the 38th Annual ACM SIGPLAN-SIGACT Symposium on Principles of Programming Languages(POPL'11). ACM, New York, NY, 535-548. DOI :http://dx.doi.org/10.1145/1926385.1926447

Hans-J. Boehm and Sarita V. Adve. 2008. Foundations of the C++ concurrency memory model. In PLDI. 68-78.

S. Borkar. 2011. The exascale challenge. In Keynote at 20th International Conference on Parallel Architectures and Compilation Techniques (PACT'11).

Zoran Budimlic, Aparna Chandramowlishwaran, Kathleen Knobe, Geoff Lowney, Vivek Sarkar, and Leo Treggiari. 2009. Multi-core implementations of the concurrent collections programming model. In IWCPC

Sebastian Burckhardt, Rajeev Alur, and Milo M. K. Martin. 2005. Verifying safety of a token coherence implementation by parametric compositional refinement. In Proceedings of VMCAI.

Lucien M. Censier and Paul Feautrier. December 1978. A new solution to coherence problems in multicache systems. IEEE Transactions on Computing C-27, 12 (December 1978), 1112-1118.

Byn Choi, Rakesh Komuravelli, Hyojin Sung, Robert Smolinski, Nima Honarmand, Sarita V. Adve, Vikram S. Adve, Nicholas P. Carter, and Ching-Tsun Chou. 2011. DeNovo: Rethinking the memory hierarchy for disciplined parallelism. In Proceedings of the 20th International Conference on Parallel Architectures and Compilation Techniques (PACT'11).

Ching-Tsun Chou, Phanindra K. Mannava, and Seungjoon Park. 2004. A simple method for parameterized verification of cache coherence protocols. In FMCAD. 382-398.

Edmund M. Clarke and E. Allen Emerson. 1982. Design and synthesis of synchronization skeletons using branching-time temporal logic. In Logic of Programs, Workshop. Springer-Verlag, London, 52-71.

David L. Dill, Andreas J. Drexler, Alan J. Hu, and C. Han Yang. 1992. Protocol verification as a hardware design aid. In ICCD’92. IEEE Computer Society, Washington, DC, 522-525.

Anwar Ghuloum, Eric Sprangle, Jesse Fang, Gansha Wu, and Xin Zhou. 2007. Ct: A Flexible Parallel Programming Model for Tera-Scale Architectures. Intel White Paper. (2007).

Stein Gjessing, Stein Krogdahl, and Ellen Munthe-Kaas. 1989. Formal Specification and Verification of SCI Cache Coherence: The Top Layers. http://citeseerx.ist.psu.edu/viewdoc/summary?doi=10.1.1.51.8390

Niklas Gustafsson. 2009. Axum: Language Overview. Microsoft Language Specification.

Stephen T. Heumann, Vikram S. Adve, and Shengjie Wang. 2013. The tasks with effects model for safe concurrency. In Proceedings of the 18th ACM SIGPLAN Symposium on Principles and Practice of Parallel Programming (PPoPP'13). ACM, New York, NY, 239-250. DOI :http://dx.doi.org/10.1145/2442516.2442540

J. Howard, S. Dighe, Y. Hoskote, S. Vangal, D. Finan, G. Ruhl, D. Jenkins, H. Wilson, N. Borkar, G. Schrom, F. Pailet, S. Jain, T. Jacob, S. Yada, S. Marella, P. Salihundam, V. Erraguntla, M. Konow, M. Riepen, G. Droege, J. Lindemann, M. Gries, T. Apel, K. Henriss, T. Lund-Larsen, S. Steibl, S. Borkar, V. De, R. Van Der Wijngaart, and T. Mattson. 2010. A 48-core IA-32 message-passing processor with DVFS in 45nm CMOS. In ISSCC. 108-109.

IntelSCC. 2009. The SCC Platform Overview. Retrieved from http://communities.intel.com/servlet/ JiveServlet/downloadBody/5 512-102-2-22524/SCC_Platform_Overview.pdf.

C. N. Ip and D. L. Dill. 1993. Efficient verification of symmetric concurrent systems. In Proceedings of the 1993 IEEE International Conference on Computer Design: VLSI in Computers and Processors (ICDD'93). 230-234. DOI : http://dx.doi.org/10.1109/ICCD.1993.393375

C. Norris Ip and David L. Dill. 1996. Better verification through symmetry. Formal Methods in System Design 9, 1 (1996), 41-75. http://dx.doi.org/10.1007/BF00625968 10.1007/BF00625968

John H. Kelm, Daniel R. Johnson, Matthew R. Johnson, Neal C. Crago, William Tuohy, Aqeel Mahesri, Steven S. Lumetta, Matthew I. Frank, and Sanjay J. Patel. 2009. Rigel: An architecture and scalable programming interface for a 1000-core accelerator. In ISCA. DOI : http://dx.doi.org/10.1145/1555754.1555774

Milind Kulkarni, Keshav Pingali, Bruce Walter, Ganesh Ramanarayanan, Kavita Bala, and L. Paul Chew. 2007. Optimistic parallelism requires abstractions. In PLDI. 211-222.

Daniel Lenoski, James Laudon, Kourosh Gharachorloo, Wolf-Dietrich Weber, Anoop Gupta, John Hennessy, Mark Horowitz, and Monica Lam. March 1992. The Stanford DASH multiprocessor. IEEE Computer 25, 3 (March 1992), 63-79.

Felix Xiaozhu Lin, Zhen Wang, Robert LiKamWa, and Lin Zhong. 2012. Reflex: Using low-power processors in smartphones without knowing them. In Proceedings of the 17th International Conference on Architectural 
Support for Programming Languages and Operating Systems (ASPLOS'12). ACM, New York, NY, USA, 13-24. DOI : http://dx.doi.org/10.1145/2150976.2150979

Jeremy Manson, William Pugh, and Sarita V. Adve. 2005. The Java memory model. In POPL.

Milo M. K. Martin, Mark D. Hill, and Daniel J. Sorin. 2012. Why on-chip cache coherence is here to stay. Commununications of the ACM 55, 7 (July 2012), 78-89. DOI : http://dx.doi.org/10.1145/2209249.2209269

Milo M. K. Martin, Daniel J. Sorin, Bradford M. Beckmann, Michael R. Marty, Min Xu, Alaa R. Alameldeen, Kevin E. Moore, Mark D. Hill, and David A. Wood. 2005. Multifacet's general execution-driven multiprocessor simulator (GEMS) toolset. SIGARCH Computer Architecture News 33, 4 (2005), 92-99. DOI : http://dx.doi.org/10.1145/1105734.1105747

Kenneth L. McMillan. 2001. Parameterized verification of the FLASH cache coherence protocol by compositional model checking. In Proceedings of the 11th IFIP WG 10.5 Advanced Research Working Conference on Correct Hardware Design and Verification Methods (CHARME'01). Springer-Verlag, London, 179195. http://dl.acm.org/citation.cfm?id=646705.702177

K. L. McMillan and Schwalbe J. 1991. Formal verification of the gigamax cache consistency protocol. In Proceedings of the International Conference on Parallel and Distributed Computing. Information Processing Society, Tokyo, Japan, 242-251.

I. Melatti, R. Palmer, G. Sawaya, Y. Yang, R. M. Kirby, and G. Gopalakrishnan. 2009. Parallel and distributed model checking in Eddy. International Journal of Software Tools Technology Transfer 11, 1 (January 2009), 13-25. DOI : http://dx.doi.org/10.1007/s10009-008-0094-x

J. M. Mellor-Crummey and M. L. Scott. April 1991. Synchronization without contention. In Proceedings of the 4th International Conference on Architectural Support for Programming Languages and Operating Systems.

A. K. Nanda and L. N. Bhuyan. 1992. A formal specification and verification technique for cache coherence protocols. In ICPP. I22-I26.

J. O'Leary, M. Talupur, and M. R. Tuttle. 2009. Protocol verification using flows: An industrial experience. In Formal Methods in Computer-Aided Design, 2009. FMCAD 2009. IEEE, 172-179. http://scholar. google.com/scholar.bib?q=info:h_Ok5nemssQJ:scholar.google.com/\&output=citation\&hl=en\&as_sdt= $2001 \&$ as_ylo $=1999 \& \mathrm{ct}=$ citation\&cd $=1$

Marek Olszewski, Jason Ansel, and Saman Amarasinghe. 2009. Kendo: Efficient deterministic multithreading in software. In ASPLOS. 97-108.

Mark S. Papamarcos and Janak H. Patel. 1984. A low-overhead coherence solution for multiprocessors with private cache memories. In Proceedings of the 11th Annual International Symposium on Computer Architecture (ISCA'84). ACM, New York, NY, 348-354. DOI : http://dx.doi.org/10.1145/800015.808204

Seungjoon Park and David L. Dill. 1995. An executable specification, analyzer and verifier for RMO (relaxed memory order). In Proceedings of the 7th Annual ACM Symposium on Parallel Algorithms and Architectures (SPAA'95). ACM, New York, NY, 34-41. DOI : http://dx.doi.org/10.1145/215399.215413

Fong Pong, Michael Browne, Andreas Nowatzyk, Michel Dubois, and Günes Aybay. 1998. Design verification of the S3.mp cache-coherent shared-memory system. IEEE Transactions on Computing 47, 1 (January 1998), 135-140. DOI : http://dx.doi.org/10.1109/12.656100

Fong Pong and Michel Dubois. 1997. Verification techniques for cache coherence protocols. ACM Computing Survey 29, 1 (March 1997), 82-126. DOI : http://dx.doi.org/10.1145/248621.248624

Jean-Pierre Queille and Joseph Sifakis. 1982. Specification and verification of concurrent systems in CESAR. In Proceedings of the 5th Colloquium on International Symposium on Programming. Springer-Verlag, London, 337-351.

Park Seungjoon and Dill David. 1996. Verification of FLASH cache coherence protocol by aggregation of distributed transactions. In Proceedings of the 8th Annual ACM Symposium on Parallel Algorithms and Architectures (SPAA'96). ACM, New York, NY, 288-296. DOI : http://dx.doi.org/10.1145/237502.237573

Hyojin Sung, Rakesh Komuravelli, and Sarita V. Adve. 2013. DeNovoND: Efficient hardware support for disciplined non-determinism. In Proceedings of the 18th International Conference on Architectural Support for Programming Languages and Operating Systems (ASPLOS'13). ACM, New York, NY, 13-26. DOI : http://dx.doi.org/10.1145/2451116.2451119

Hyojin Sung, Rakesh Komuravelli, and Sarita V. Adve. 2014. DeNovoND: Efficient hardware for disciplined nondeterminism. Micro, IEEE 34, 3 (May 2014), 138-148. DOI : http://dx.doi.org/10.1109/MM.2014.5

Mohsen Vakilian, Danny Dig, Robert Bocchino, Jeffrey Overbey, Vikram Adve, and Ralph Johnson. 2009. Inferring method effect summaries for nested heap regions. In Proceedings of the 2009 IEEE/ACM International Conference on Automated Software Engineering (ASE'09). IEEE Computer Society, Washington, DC, 421-432. DOI : http://dx.doi.org/10.1109/ASE.2009.68

Dana Vantrease, Mikko H. Lipasti, and Nathan Binkert. 2011. Atomic coherence: Leveraging nanophotonics to build race-free cache coherence protocols. In Proceedings of the 2011 IEEE 17th International 
Symposium on High Performance Computer Architecture (HPCA'11). IEEE Computer Society, Washington, DC, 132-143.

Gwendolyn Voskuilen and T. N. Vijaykumar. 2014. High-performance fractal coherence. In Proceedings of the 19th International Conference on Architectural Support for Programming Languages and Operating Systems (ASPLOS'14). ACM, New York, NY, 701-714. DOI : http://dx.doi.org/10.1145/2541940.2541982

WoDET. 2009. Workshop on determinism and correctness in parallel programming.

WoDET. 2011. Workshop on determinism and correctness in parallel programming.

WoDET. 2012. Workshop on determinism and correctness in parallel programming.

WoDET. 2013. Workshop on determinism and correctness in parallel programming.

Jason Zebchuk, Vijayalakshmi Srinivasan, Moinuddin K. Qureshi, and Andreas Moshovos. 2009. A tagless coherence directory. In MICRO.

Meng Zhang, Jesse D Bingham, John Erickson, and Daniel J. Sorin. 2014. PVCoherence: Designing flat coherence protocols for scalable verification, In Proceedings of the 2014 IEEE International Symposium on High Performance Computer Architecture. HPCA (2014), 1-12.

Meng Zhang, Alvin R. Lebeck, and Daniel J. Sorin. 2010. Fractal coherence: Scalably verifiable cache coherence. In Proceedings of the 2010 43rd Annual IEEE/ACM International Symposium on Microarchitecture (MICRO'43). IEEE Computer Society, Washington, DC, 471-482. DOI : http://dx.doi.org/10.1109/ MICRO.2010.11

Hongzhou Zhao, Arrvindh Shriraman, Snehasish Kumar, and Sandhya Dwarkadas. 2013. Protozoa: Adaptive granularity cache coherence. In Proceedings of the 40th Annual International Symposium on Computer Architecture (ISCA'13). ACM, New York, NY, 547-558. DOI : http://dx.doi.org/10.1145/2485922.2485969

Received May 2014; accepted August 2014 